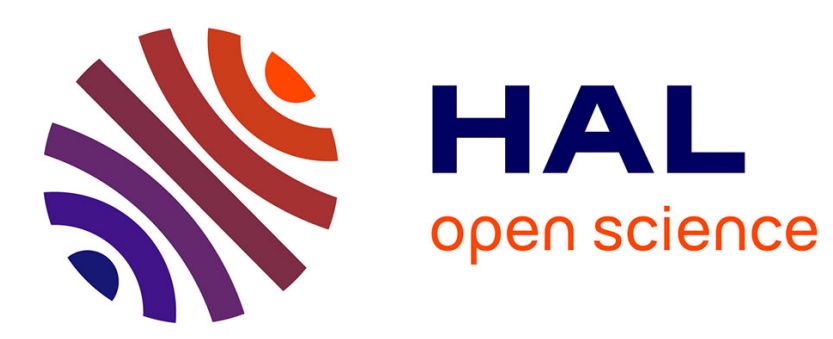

\title{
Can an Underactuated Leg with a Passive Spring at the Knee Achieve a Ballistic Step?
}

Bernard Espiau, Isabelle Guigues, Roger Pissard-Gibollet

\section{To cite this version:}

Bernard Espiau, Isabelle Guigues, Roger Pissard-Gibollet. Can an Underactuated Leg with a Passive Spring at the Knee Achieve a Ballistic Step?. RR-3544, INRIA. 1998. inria-00073140

\section{HAL Id: inria-00073140 \\ https://hal.inria.fr/inria-00073140}

Submitted on 24 May 2006

HAL is a multi-disciplinary open access archive for the deposit and dissemination of scientific research documents, whether they are published or not. The documents may come from teaching and research institutions in France or abroad, or from public or private research centers.
L'archive ouverte pluridisciplinaire $\mathbf{H A L}$, est destinée au dépôt et à la diffusion de documents scientifiques de niveau recherche, publiés ou non, émanant des établissements d'enseignement et de recherche français ou étrangers, des laboratoires publics ou privés. 
INSTITUT NATIONAL DE RECHERCHE EN INFORMATIQUE ET EN AUTOMATIQUE

\section{Can an Underactuated Leg with a Passive Spring at the Knee Achieve a Ballistic Step?}

Bernard Espiau, Isabelle Guigues, Roger Pissard-Gibollet

\section{$N^{\circ} 3544$}

Novembre 1998

THÈME 4 



\title{
Can an Underactuated Leg with a Passive Spring at the Knee Achieve a Ballistic Step?
}

\author{
Bernard Espiau, Isabelle Guigues, Roger Pissard-Gibollet \\ Thème 4 - Simulation et optimisation \\ de systèmes complexes \\ Projet BIP
}

Rapport de recherche $\mathrm{n}^{\circ} 3544$ - Novembre 1998 - 23 pages

\begin{abstract}
In this paper, we present the design and the results of a control scheme aimed at moving an underactuated double pendulum simulating a leg. The system is actuated at the hip and includes a spring at the knee. It interacts with the grou nd, which is in fact a treadmill, through a telescopic foot made of a linear spri ng and a damper. The control scheme is different in the 2 phases, swing and stance, and a repetitive step is achieved by switching between these two controls. The paper describes the used models, the control algorithms and their implementation. It pr esents also experimental results of the approach.
\end{abstract}

Key-words: Underactuated systems, Ballistic Motion, Springs, Biped Robots 


\section{Commande d'une jambe sous-actionnée munie d'un ressort au genou}

Résumé : Cet article décrit la modélisation et la commande d'un double pendule sousactionné simulant une jambe humaine. Seule la hanche est munie d'un moteur, tandis que le genou comporte un rappel élastique analogue à celui de certaines orthèses destinées à compenser l'absence de fléchisseurs en orthopédie. Le contact avec le sol se fait au travers d'un pied télescopique comportant un ressort et un amortisseur. Enfin, un tapis roulant permet d'entraîner le pied, donc de simuler la marche cyclique. La commande est du type "linéarisation partielle" et est différente selon la phase considérée (support ou balancement). Le pas est ainsi obtenu en combinant un mouvement balistique garantissant la nonpénétration du pied et le maintien en extension de l'ensemble pendant le support. L'article décrit la commande proposée, implémentée sous ORCCAD, et donne quelques résultats expérimentaux.

Mots-clé : Mécanique, Cinématique, Robotique, Sous-actionnement, Pendule 


\section{Introduction}

The remote origin of this work is a medical application. In a way similar to [1], the problem we addressed was to design a reconfigurable knee orthosis with tunable passive springs and dampers. This orthosis was aimed at improving rehabilitation programs following an operation and helping patients or elderly having weak flexion or extension capabilities ([3]). An effect of the system is that, when walking, a patient who wears such a knee-pad may have to use dynamical (inertial) effects together with his own remaining actuation capabilities in order to counter the spring force and reach the quasi-full leg extension which is required for starting the stance phase.

In complement to classical experimental protocols to be conducted on patients in a hospital, we therefore found interesting to experimentally investigate the purely mechanical and control issues of the problem on a dedicated robotic device. We thus designed and realized a double pendulum, with size and mass repartition close to human's ones and having a single actuator at the hip and a spring at the knee in order to study the synthesis of gaits for this system. Compared to a human or to a classical biped robot, further difficulty with a vertical planar double pendulum arises, because the hip position is fixed with respect to the ground. In order to simulate the motion, we therefore added a treadmill to the setup and mounted a telescopic foot using a second linear spring.

And, finally, we designed the control. Since dynamics issues were strongly involved and because of the existence of different phases in the step, the system was considered as an hybrid one (i.e mixing continuous-time and discrete-events) with particular real-time requirements. This led us to take a special care to the control implementation. The purpose of this paper is therefore to present the control scheme we propose and to report the obtained results. Section 2 gives a brief review of existing works; section 3 presents the general approach we used, which is based on partial feedback linearization and projection; in section 4 , we describe the experimental setup and the related models. Section 5 present the control algorithms; we also give a few words on their implementation through ORCCAD (cf [19]). Finally we present the results (section 6), which are experimental ones, simulation being not very relevant in the present case.

\section{A Brief Tour of the Literature}

The present paper adresses simultaneously questions of ballistic walking, underactuation and effects of springs on the gait. Without claiming to perform an exhaustive survey, we can nevertheless present a quick review of some existing works in these different fields. One of the early references in human ballistic walking is [9], where the proposed dynamical model assumes the quasi-absence of muscular moments during the swing phase. The further effect of non-free orthoses at the knee on the walk is studied in [1, 2, 3]. However, the literature on that point does not seem very extensive. On the contrary, the use of springs in robot joints have soon been recognized as an interesting way of realizing energy storage or impact absorption and many works have been reported in the literature. Although run-

$\operatorname{RR} n^{\circ} 3544$ 
ning or hopping robots be obviously firstly concerned by this approach (see $[4,15]$ and, more generally, the work done in the MIT LegLab ${ }^{1}$ ), the case of walking machines is also considered (cf. [5, 6] and Pratt's turkeys [14]).

In the domain of automatic control, underactuated systems have been rather largely investigated. As an interesting class of nonlinear systems the tangent linear approximation of which is not controllable, underactuated robots, like flexible or non-holonomic ones, have been soon targeted by researchers from the automatic control area. For example, [11, 10, 12] consider planar robots with joint elasticities; more generally, [13] studies robotics systems with 2nd order non-holonomic constraints. The capacity of controlling underactuated robots using their dynamical coupling characteristics is modelled in $[7,8]$. Finally, let us specially mention interesting works around Acrobot-like systems (these are 2 dof robots, with a single actuator either at the hip - the Pendubot - or at the knee, and submitted to the gravity, contrary to previous cases): [16] proposes a decoupling and partial feedback linearization scheme with control switching; [17] uses two control schemes for a hopping Acrobot, one for the flight phase and the other one in the stance phase. A last point to be mentionned is the fact that the active control of a compass robot using the hip actuator as done in [20] is a problem which can be viewed as a special case of control of an underactuated double pendulum.

In fact, and to our knowledge, the problem addressed in this paper has not been considered under the same form in the literature. Thus, although some parts of the control we propose are not really new, the global solution we bring to the overall problem of achieving a ballistic step with an underactuated double pendulum having a knee spring seems to be original.

\section{Background in Modelling and Control}

\subsection{Unconstrained case}

Let us consider a robot with rigid links, $n$ joints and a fixed basis. Let us suppose that the dimension of the actuation space (assumed to be of invariant structure) is $m<n$. By performing a change of variables if needed, its dynamical equation may always be written under the form

$$
M(q) \ddot{q}+N(q, \dot{q})=B \Gamma=\left(\begin{array}{c}
\Gamma \\
0
\end{array}\right)
$$

where $q$ is a parametrization of the configuration space (in general the set of joint variables, but this is not always the case, especially for biped robots), $M(q)$ the s.d.p. matrix of kinetics energy and $N(q, \dot{q})=N^{\prime}(q, \dot{q})+G(q) . N^{\prime}$ is the vector of Coriolis/centrifugal forces and $G$ the vector of potential-based forces. $\Gamma$ is the $m$-dimensional actuation vector.

${ }^{1}$ http://www.leglab.ai.mit.edu/leglab 


\section{Remark}

The section title, "Unconstrained case", may appear as ambiguous, since the lower part of (1) (the zero-dynamics) can also be viewed as a non-holonomic constraint associated with the actuated part of the system. We therefore restrict the use of the term "constraint" in the following to holonomic ones, which confines the evolution of the system to a submanifold of the configuration space.

The set of configuration points $q^{*}$ that we can try to stabilize is given by the equilibrium manifold of (1): $G_{2}\left(q^{*}\right)=0$ where $G_{2}$ is the lower part of $G$ corresponding to the non-actuated part $\left(G_{2}\right.$ includes all potential-based forces). We already see that the most favourable situation is the one of a planar horizontal robot without joint elasticities, where the equilibrium manifold is the whole configuration space. This is not our case, since the double pendulum is submitted to gravity and has a spring at the knee joint.

Let us now define a $m$-dimensional output function $e(q, t)$ to be regulated to zero as an user specification. Differentiating $e$ twice gives

$$
\ddot{e}=J \ddot{q}+f(q, \dot{q}, t)
$$

where $J$ is the jacobian matrix of $e$ w.r.t $q$. Combining (1) and (2) gives the reduced dynamics in the output space:

$$
\ddot{e}=J M^{-1}\left(\begin{array}{c}
\Gamma \\
0
\end{array}\right)-J M^{-1} N+f
$$

Let us now partition $M$ as $\left(\begin{array}{ll}M_{11} & M_{12} \\ M_{12}^{T} & M_{22}\end{array}\right)$ with $\operatorname{dim}\left(M_{11}\right)=m \times m$. It is easy to see that (3) may be written as

$$
\ddot{e}=R K^{-1} \Gamma-J M^{-1} N+f
$$

where

$$
\begin{gathered}
R=J_{1}-J_{2} M_{22}^{-1} M_{12}^{T} \\
K=M_{11}-M_{12} M_{22}^{-1} M_{12}^{T}
\end{gathered}
$$

with $J=\left(J_{1}(m \times m) J_{2}\right)$. Assuming that $M_{22}, R$ and $K$ are nonsingular matrices, we can therefore finally write

$$
\Gamma=E \ddot{e}+F
$$

where $E=K R^{-1}$ and $F=K R^{-1} J_{2} M_{22}^{-1} N_{2} f+N_{1}-M_{12} M_{22}^{-1} N_{2}$ with $N=\left(\begin{array}{c}N_{1} \\ N_{2}\end{array}\right)$

Finally, and in a way similar to [16]), a possible PD-type control scheme is

$$
\Gamma=\hat{E}\left(-K_{p} e-K_{v}\left(J \dot{q}+\frac{\partial e}{\partial t}\right)\right)+\hat{F}
$$

This control achieves an approximate partial linearization and decoupling: approximate, because the hats on the terms in (8) indicate that the models used in the control may differ 
from the theoretical expressions; partial since the linearization/decoupling concerns only the $m$-dimensional controlled manifold while the complementary one evolves freely. We will use a control belonging to this class in the swing phase of our double pendulum (see section 5).

\subsection{Constrained case}

It is generally possible to model a biped robot in the swing phase as an open articulated chain , satisfying therefore the equations of the previous section. During the stance phase, new constraints appear, which can be modelled either as unilateral (see [22]) or, more often as bilateral. Therefore, the model has to be modified in order to incorporate the constraints in the following way (see full developments in [21]).

Let us complete (1) with the constraints equation

$$
h(q)=0
$$

. We set $\operatorname{dim}(h)=p<n$, with $n-p=n_{1}$, and we further assume that the constraints are compatible, i.e. the solution of (9) is not an empty set, and independent, i.e

$$
\operatorname{rank}\left(C=\frac{\partial h}{\partial q}\right)=p
$$

The constrained dynamic equation can now be written as

$$
M(q) \ddot{q}+N(q, \dot{q})=B \Gamma-C^{T}(q) \lambda
$$

where $\lambda$ is the $p$-dimensional array of Lagrange multipliers associated with the constraints. Differentiating twice the equation (9) gives

$$
C \ddot{q}+s(q, \dot{q})=0
$$

The equation (11) can also be written as

$$
\ddot{q}=M^{-1} B \Gamma-M^{-1} N-M^{-1} C^{T} \lambda
$$

Using this result in (12) gives:

$$
\lambda=\left(C M^{-1} C^{T}\right)^{-1}\left(C M^{-1}(B \Gamma-N)+s\right)
$$

Replacing in (11) leads to:

$$
\ddot{q}=P M^{-1}(B \Gamma-N)-C^{\dagger} s
$$

where

$$
C^{\dagger}=M^{-1} C^{T}\left(C M^{-1} C^{T}\right)^{-1}
$$

and with the notation

$$
P=I_{n}-C^{\dagger} C
$$


$P, n \times n$, is a projection operator onto the null space of $C$ with respect to the kinetics metrics $M$ (this means that we have $P^{T} M P=M P$ instead of $P^{T} P=P$ as involved by the euclidean metrics). Its rank is $n-p=n_{1}$. Multiplying equation (15) by $P$ and using the properties: $P^{2}=P$ and $P C^{\dagger}=0$ gives finally the equations of the constrained dynamics:

$$
P\left(\ddot{q}-M^{-1}(B \Gamma-N)\right)=0 ; h(q)=0
$$

The LHS of equation (18) is of dimension $n$. Nevertheless, the rank of $P$ is $n_{1}$. We can therefore replace (18) by a set of $n_{1}$ equations in the following way: (18) means that the projection is $M$-orthogonal to $N S$, the null space of $C$. Therefore, the projected vector is $M$-orthogonal to any set of basis vectors of $N S$. Let us partition $C$ as

$$
C=\left(\begin{array}{ll}
C_{1} & C_{2}
\end{array}\right)
$$

where $C_{1}$ is $p \times p$, assumed to be nonsingular. We can therefore choose as a basis of $N S$ the columns of the $n_{1} \times n$ matrix:

$$
\bar{R}=\left(\begin{array}{c}
-C_{1}^{-1} C_{2} \\
I_{n_{1}}
\end{array}\right)^{T}
$$

(It can be easily verified that $C \bar{R}^{T}=0$ ). Finally, the $n$ equations describing the constrained dynamics can be defined as the set:

$$
\left\{\begin{array}{l}
\bar{R}(M \ddot{q}-B \Gamma+N)=0 \\
h(q)=0
\end{array}\right.
$$

The number of degrees of freedom left available for moving the system (21) is $n_{1}$. We can therefore specify again our control goal as the regulation at zero of a desired output function of dimension $n_{1}, e(q, t)$. Combining (2) with (12) gives

$$
\ddot{q}=\bar{K}\left(\begin{array}{c}
s \\
\ddot{e}-f
\end{array}\right)
$$

where

$$
\bar{K}=\left(\begin{array}{c}
C \\
J
\end{array}\right)^{-1}
$$

For $\bar{K}$ to be nonsingular, the independency of $e$ and $h$ is required. Let us now partition $\bar{K}$ as

$$
\bar{K}=\left(\begin{array}{ll}
\bar{K}_{1} & \bar{K}_{2}
\end{array}\right)
$$

where $\bar{K}_{1}$ is $n \times p$ and $\bar{K}_{2}$ is $n \times n_{1}$, both assumed to be of full rank. Using (24) and (22) in (21) finally leads to the dynamics equation in the output space:

$$
M^{\prime}(q) \ddot{e}+f^{\prime}(q, \dot{q}, t)=u
$$

where

$$
M^{\prime}=\bar{R} M \bar{K}_{2},
$$


assumed to be nonsingular,

$$
f^{\prime}=\bar{R} M\left(\bar{K}_{1} s-\bar{K}_{2} f\right)+\bar{R} N
$$

and

$$
u=R B \Gamma
$$

Now, a decoupling and feedback linearizing control is given by

$$
u=M^{\prime}(q)\left(-K_{p} e-K_{v}\left(J \dot{q}+\frac{\partial e}{\partial t}\right)\right)+f^{\prime}(q, \dot{q}, t)
$$

where $K_{p}$ and $K_{v}$ are diagonal positive matrices. This ideal control ensures a linear secondorder decoupled behavior for $e$. Given $u$, it remains to compute the actuator torques, $\Gamma$, the dimension of which can be greater than $n_{1}$. This can be done by selecting, for example, the torques which minimize some energy-based criterion, or the ones which ensure that unilateral constraints will not be violated (see [22]). We will use a control of type $(28,29)$ simplified in the stance phase of our double pendulum.

\section{The Experimental Setup}

The double pendulum (see pictures in section 6) includes a thigh (length $0.45 \mathrm{~m}$, adjustable mass up to $6 \mathrm{~kg}$ ), a leg (length $0.45 \mathrm{~m}$, adjustable mass up to $4 \mathrm{~kg}$ ) and a telescopic foot made of a spring/damper system with a range of $4 \mathrm{~cm}$. The effective contact area (when the leg is vertical) is at most of $2 \mathrm{~cm}^{2}$. The system is equipped with a spring at the knee (see figure 2) and can touch a treadmill, the maximum speed of which is $25 \mathrm{~cm} / \mathrm{s}$.

The actuation is realized by a Parvex Motor (nominal torque 2 N.m) with a gear providing with a reduction ratio of 25 . Encoders measure angular positions of the two joints.

\subsection{Model in the Swing Phase}

The notation is given in figures 2 and 1 . The configuration vector is $q=\left(q_{1}, q_{2}\right)$. In the following, $c_{i}$ and $s_{i j}$ stand for $\cos \left(q_{i}\right)$ and $\sin \left(q_{i}+q_{j}\right)$ respectively. In equation (1) the inertia matrix for the pendulum is

$$
\begin{aligned}
& M_{11}=\frac{1}{4} m_{1} l_{1}^{2}+m_{2}\left(l_{1} l_{2} c_{2}+l_{1}^{2}+\frac{1}{4} l_{2}^{2}\right)+I_{1}+I_{2} \\
& M_{12}=M_{21}=m_{2}\left(\frac{1}{2} l_{1} l_{2} c_{2}+\frac{1}{4} l_{2}^{2}\right)+I_{2} \\
& M_{22}=\frac{1}{4} m_{2} l_{2}^{2}+I_{2}
\end{aligned}
$$

The Coriolis and Centrifugal term is given by $N^{\prime}(q, \dot{q})=Q(q, \dot{q}) \dot{q}$ where

$$
\begin{aligned}
& Q_{11}=-\frac{1}{2} m_{2} l_{1} l_{2} s_{2} \dot{q}_{2} \\
& Q_{12}=Q_{11}\left(\dot{q}_{1}+\dot{q}_{2}\right) \\
& Q_{21}=-Q_{11} \\
& Q_{22}=0
\end{aligned}
$$




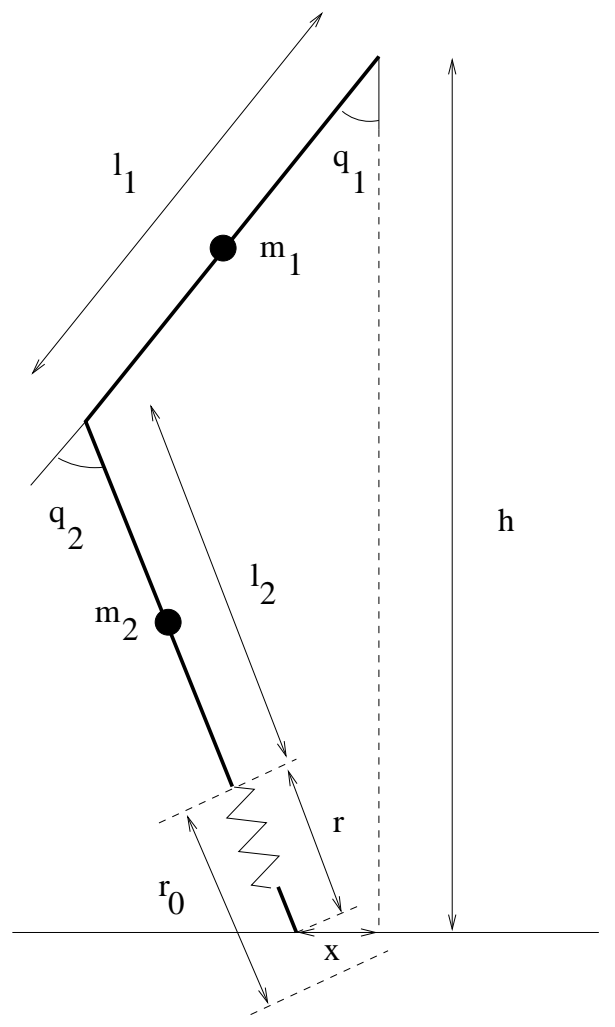

Figure 1: Scheme of the Double Pendulum in Stance Phase

The gravity vector is

$$
G^{1}=-g\left(\begin{array}{c}
m_{1} l_{1} s_{1}-m_{2}\left(\frac{1}{2} l_{2} s_{12}+l_{1} s_{1}\right) \\
m_{2} l_{2} s_{12}
\end{array}\right)
$$

The torque exerted by the spring at the knee (cf figure 2) is

$$
G^{2}=\left(\begin{array}{c}
0 \\
K\left(l-l_{0}\right) l_{\perp}
\end{array}\right)
$$

with

$$
\begin{gathered}
l=\sqrt{\left(a_{2} c_{2}+a_{1}\right)^{2}+\left(a_{2} s_{2}-b\right)^{2}} \\
l_{\perp}=\sqrt{a_{1}^{2}+b^{2}-\frac{1}{4 l^{2}}\left(l_{2}+a_{1}^{2}+b^{2}-a_{2}^{2}\right)^{2}}
\end{gathered}
$$

and the term $G$ in (1) is given by $G=G^{1}+G^{2}$. Finally, let us note that $\Gamma$ is here the 1D hip actuator torque.

$\mathrm{RR} \mathrm{n}^{\circ} 3544$ 


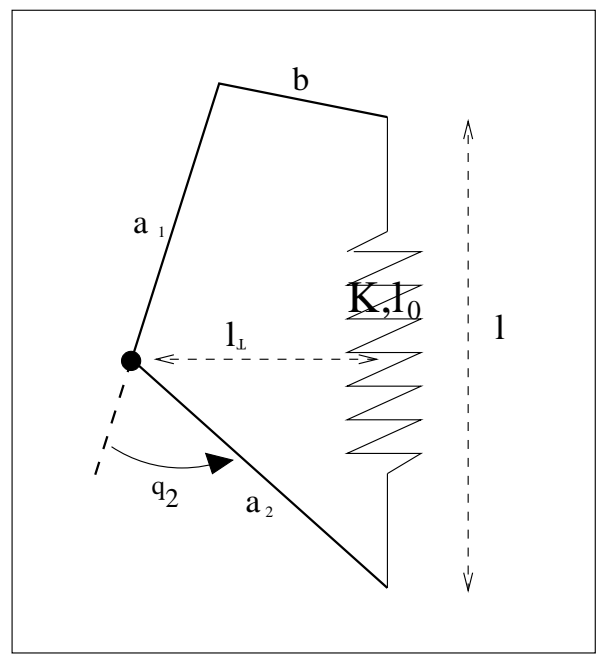

Figure 2: Principle of setting-up a spring at the knee

\subsection{Model in the Stance Phase}

We assume at this stage that the foot spring is in the leg direction and that its mass and inertia can be neglected. We also suppose that the normal contact force and the friction coefficient are large enough to avoid sliding and lifting of the foot. Note that, clearly, these asumptions are not true at the beginning and the end of the stance phase. However, we will use them in order to derive the control, then we will experimentally verify that we nevertheless can cope with the cases where they are not satisfied.

Now, the configuration space of the system is of dimension 3: $\left\{q_{1}, q_{2}, r\right\}$, since it should include the spring length variable. The 2-dimensional constraint, modelled as bilateral, writes:

$$
\left\{\begin{array}{l}
l_{1} c_{1}+\left(l_{2}+r\right) c_{12}=h \\
l_{1} s_{1}+\left(l_{2}+r\right) s_{12}=x(t)
\end{array}\right.
$$

Therefore, the system is no more underactuated in this phase. The couple (36) can also be written as

$$
\left\{\begin{array}{l}
r=\frac{h-l_{1} c_{1}}{c_{12}} \quad(a) \\
l_{1} s_{1}+h-l_{1} c_{1} t_{12}-x(t)=0(b)
\end{array}\right.
$$

The equation (37) (a) can be taken as the constraint (9) and the related dynamics, (11), is now the 2-dimensional system:

$$
M(q) \ddot{q}+N^{\prime}(q, \dot{q})+G^{1}(q)+G^{2}(q)+K^{\prime}(q)=\left(\begin{array}{c}
\Gamma \\
0
\end{array}\right)-C^{T}(q) \lambda
$$


where

$$
K^{\prime}(q)= \begin{cases}k\left(r-r_{0}\right) l_{1} s_{2} & \text { for } r<r_{0} \\ 0 & \end{cases}
$$

with $K(q)=0$ for $r \geq r_{0}, r$ being given in (37) (a), and $C^{T}$ is the jacobian matrix of the constraint (37) (b):

$$
C^{T}=\left(\begin{array}{c}
C^{1} \\
C^{2}
\end{array}\right)=\left(\begin{array}{c}
l_{1} c_{1}+l_{1} s_{1} t_{12}+\frac{h-l_{1} c_{1}}{c_{12}^{2}} \\
\frac{h-l_{12} c_{1}}{c_{12}^{2}}
\end{array}\right)
$$

\section{Control}

\subsection{Control in the Swing Phase}

In that phase, our objective is to have the leg almost fully extended (without further knee angle increase), despite gravity, spring and inertia effects, at a desired value of the hip angle, and after a given time. The initial conditions are the ones of the free system in equilibrium. We therefore simply define as the task function to regulate:

$$
e_{1}(q, t)=q_{1}(t)-q_{1}^{d}(t), t \in[0, T]
$$

with $q_{1}(0)=q_{1}^{d}(0)$ and $\dot{q_{1}^{d}}(0)=\dot{q}_{1}^{d}(T)=0$. We also need to ensure that $\dot{q}_{2}(T)=q_{2}(T)=0$ It remains now to find an adequate trajectory, $q_{1}^{d}(t)$, satisfying the expressed goals. We will use the following result ${ }^{2}$

Proposition: For the system $(1,30,31,32,33)$, if the joint velocities are bounded, there exists $\Theta, \frac{\pi}{2} \leq$ $\Theta<\pi$ such that $\forall\left|q_{2}\right|<\Theta$, there exists $\ddot{q}_{1}{ }^{m}>0$ such that $\forall\left|\ddot{q}_{1}\right|>\ddot{q}_{1}{ }^{m}$, then sign $\left(\ddot{q}_{2}\right)=$ $-\operatorname{sign}\left(\ddot{q}_{1}\right)$.

The proof is extremely simple and given in the appendix.

In fact, using this property will allow us to search for the final acceleration of $q_{1}$ able to place $q_{2}$ at the right value by inertia coupling effects only ${ }^{3}$. The easiest way of finding the desired trajectory $q_{1}^{d}(t)$ is to compute a polynomial satisfying the constraints:

$q_{1}^{d}(0)=q_{1}(0)$ (measured); $\dot{q}_{1}^{d}(0)=0 ; q_{1}^{d}(T)=q_{1}^{*} ; \dot{q}_{1}^{d}(T)=0 ; \ddot{q}_{1}^{d}(T)=\omega$. Given $T$ and $q_{1}^{*}, \omega$ is our single tuning parameter, and the polynomial is $q_{1}^{d}(t)=q_{1}(0)+\sum_{i=1}^{4} a_{i} t^{i}$, where

$$
\begin{array}{r}
a_{1}=0 \\
a_{2}=\left(\omega+6 \frac{\delta}{T^{2}}\right) / T^{2} \\
a_{3}=-\left(\omega+8 \frac{\delta}{T^{2}}\right) / T \\
a_{4}=\left(\frac{\omega}{2}+8 \frac{\delta}{T^{2}}\right) / T^{2}
\end{array}
$$

\footnotetext{
${ }^{2}$ which can be related in some sense to Bergerman's actuability analysis ([8]).

${ }^{3}$ This approach will therefore lead to have $q_{2}$ reaching its extremal value with a slight time delay after $T$, which in practice is not a significative drawback.
} 
with $\delta=q_{1}^{*}-q_{1}(0)$.

To complete the control equations, it remains to choose the models to be used in (8). In order to ensure a better tracking of the reference trajectory, we chose to compensate for the gravity term $G^{1}(q)$ and for the acceleration of the reference trajectory. Knowing that, here, the matrix $R$ of (5) is equal to 1 , the final control scheme is:

$$
\Gamma_{1}=\hat{K}\left(-K_{p} e-K_{v}\left(\dot{q}_{1}-\dot{q}_{1}^{d}\right)\right)+G^{1}(q)+\hat{M}_{11} \ddot{q}_{1}^{d}(t)
$$

In this expression, the inertia matrix (therefore $\hat{K}$ and $\hat{M}_{11}$ ) is taken constant; the spring torque, assumed unknown, and the coriolis/centrifugal term are not compensated for.

\subsection{Control in the Stance Phase}

We simply take here

$$
e_{2}=q_{2}
$$

and apply the control of section 3.2 using eq. (38) and (40). The matrix $\bar{R}$ of (21) is then

$$
\bar{R}=\left(\begin{array}{ll}
-C^{1} / C^{2} & 1
\end{array}\right)
$$

and $\bar{K}(24)$ is

$$
\bar{K}=\left(K_{1} K_{2}\right)=\frac{1}{C^{1}}\left(\begin{array}{cc}
1 & -C^{2} \\
0 & 1
\end{array}\right)
$$

Note that, here, we have $\bar{R}=K_{2}^{T}$. The expression (28) writes as:

$$
u=-\frac{C^{2}}{C^{1}} \Gamma
$$

and the final control (eqs. (25 to 29)) is:

$$
\Gamma_{2}=\hat{A}\left(-K_{p} q_{2}-K_{v} \dot{q}_{2}\right)+\hat{B}
$$

where $\hat{A}$ and $\hat{B}$ are suitable approximations for

$$
A=-C^{1} / C^{2} K_{2}^{T} M K_{2}
$$

and

$$
B=K_{2}^{T} N
$$

respectively. Let us now consider the case where $q_{2}$ is small, which is required for having the leg extended and thus a stance phase long enough. Then, $M(q)$ is approximately constant and also:

$$
\begin{array}{r}
r \approx\left(\frac{h}{c_{1}}-l_{1}\right)-l_{2} \\
C^{1} \approx l_{1} c_{1}+C^{2} \\
C^{2} \approx \frac{1}{c_{1}}\left(r+l_{2}\right)
\end{array}
$$


Now, if the range of variation of $q_{1}$ is small (in the stance phase, for maintaining the contact, $\left|q_{1}\right|$ cannot be greater than 7 deg.), we can set $c_{1} \approx 1$. We can therefore choose $\hat{A}=A_{\left(q_{1}=q_{2}=0\right)}$ in (51). Since the gravity effects are small when the leg is almost vertical and that the velocities are small because they are only due to the treadmill motion, we can simply take $\hat{B}=0$ in (51). We have constated experimentally that reasonnable values of gains allow to compensate for the influence of these modelling approximations on the tracking error.

\subsection{Control Implementation}

In order to achieve a succession of full steps, the two controls have to be chained in an endless loop. Transition from swing to stance controls is triggered by the reaching of the full extension while the reverse transition requires the detection of the "toe off" event. The early activation of the stance control (i.e before the heel contact) is required for adding a supplementary torque on the hip aimed at maintaining the extension despite the spring effect. In fact, if we let the leg swing down freely under the gravity only, the knee flexes and the leg misses the ground.

Several other events have to be handled during the motion in order to achieve the step safely: the exerted torques and the joint angles should be bounded, the phase durations are submitted to time limits (watchdogs), etc... In order to handle properly such events and to ensure that real time constraints are well satisfied, as required by the process dynamics, we used the ORCCAD ([19]) environment. ORCCAD ${ }^{4}$ is a software environment dedicated to the design (specification, programming) and the implementation (automatic code generation) of advanced robotics control systems. It is based on two main entites: the Robot-task, which is a kind of elementary action where the designer describes the control law under the form of communicating modules and its internal scheduling through an implicit automaton; the Robot-procedure which allows to logically organize and schedule all the required elementary actions from their abstract view. Figures (3) and (4) show the stance and swing robot-tasks respectively, and figure (5) shows the scheduling code of the robot-procedure step, which is automatically generated. The execution code, running under the Vx Works Real-Time OS is also automatically generated.

\section{Results}

The control laws have been implemented with a hip extension angle $q_{1}^{*}$ of 20 deg and a swing time $T$ of $1.2 \mathrm{sec}$. The treadmill velocity varied from $1 \mathrm{~cm} / \mathrm{sec}$ to $25 \mathrm{~cm} / \mathrm{sec}$. The system then undergone successive regular cycles, achieving therefore successfully the goal of ballistic walking. Figure (6) shows the evolution of the joint variables during the swing phase, including a 2 sec. initializing motion. In this figure, the vertical axis unit is 1 radian, and the total range coverd by the horizontal axis is $3.5 \mathrm{sec}$. Here, the maximum value of

\footnotetext{
${ }^{4}$ http://www.inrialpes.fr/iramr/pub/Orccad/Presentation/frame-eng.html
} 


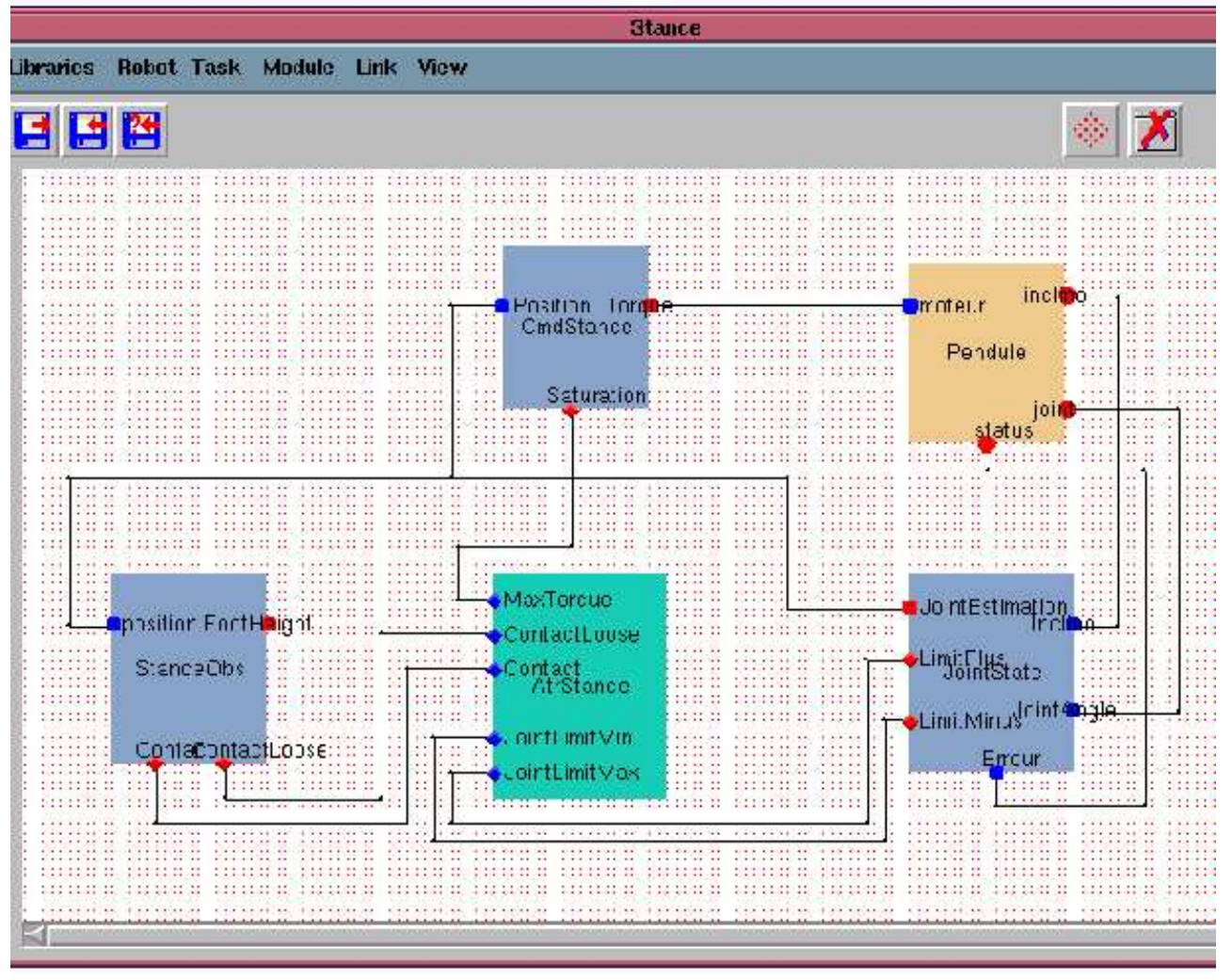

Figure 3: The robot-task "stance"

INRIA 


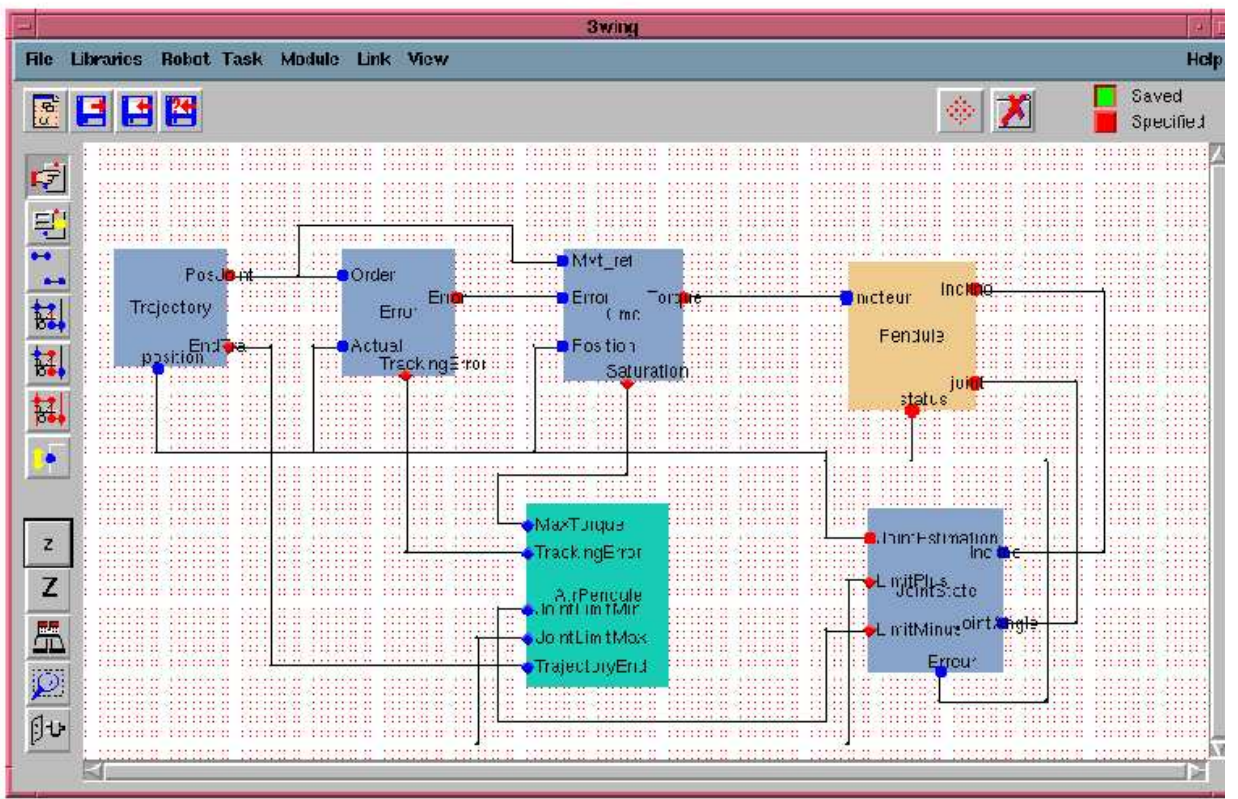

Figure 4: The robot-task "swing"

$\mathrm{RR} \mathrm{n}^{\circ} 3544$ 


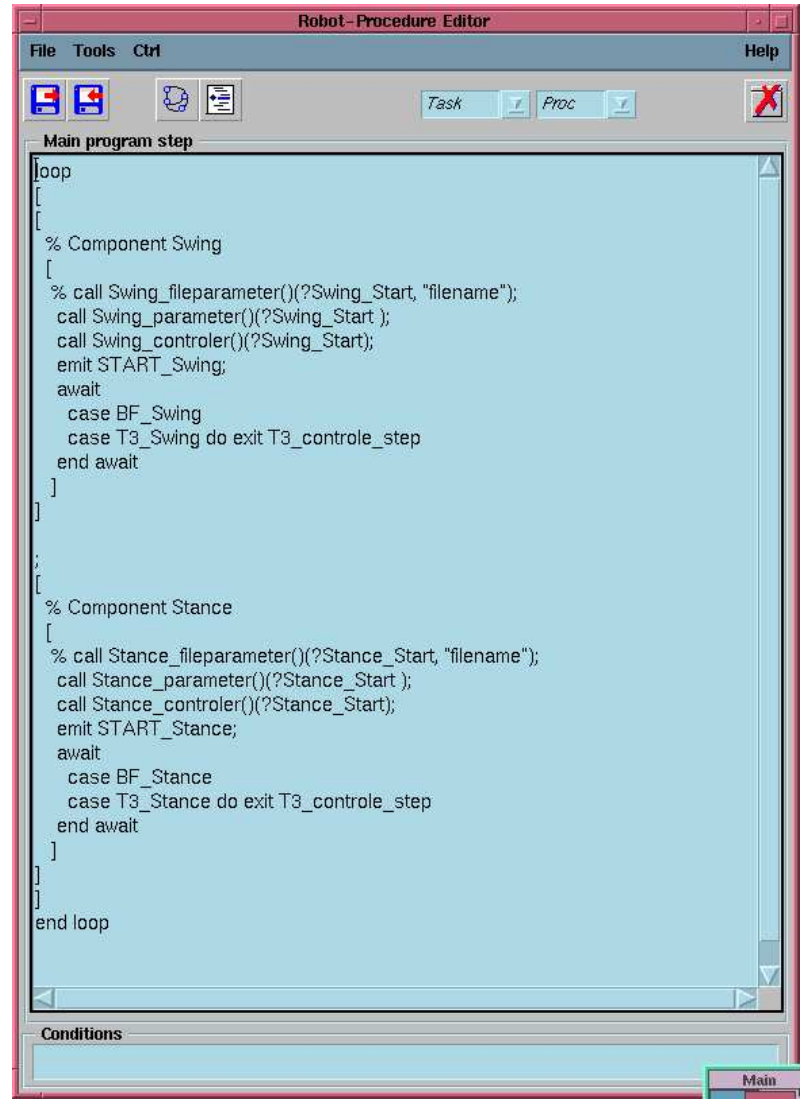

Figure 5: The robot-procedure "step" 
$q_{2}$ is reached immediatly after the last plotted point... We have experimentally constated on these records that the influence of $\omega$ on the extremal value of $q_{2}$ was the one expected. More precisely, its variation with respect to that of $\omega$ was monotonic within a large range. Note also that, here, the part of the trajectory resulting from the polynomial which would have led to a small back motion is replaced by a constant value.

Finally, we present, in figures 7 to 12 , six snapshots of a full step, showing the efficiency of the control scheme.

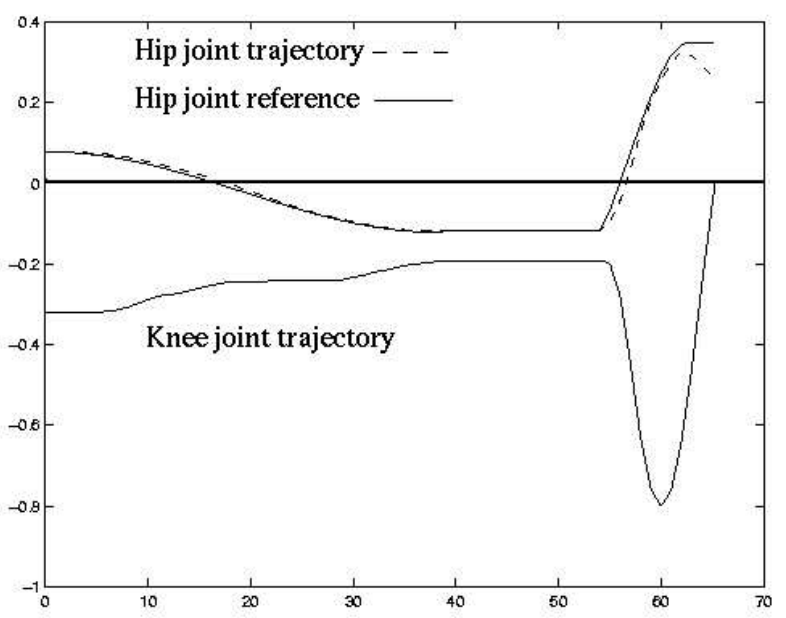

Figure 6: Trajectories of joint variables during the swing phase

$\mathrm{RR} \mathrm{n}^{\circ} 3544$ 


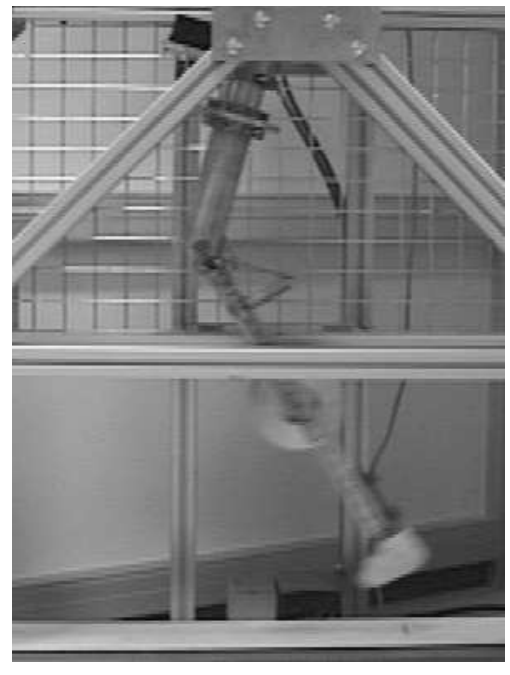

Figure 7: Step: Mid Swing

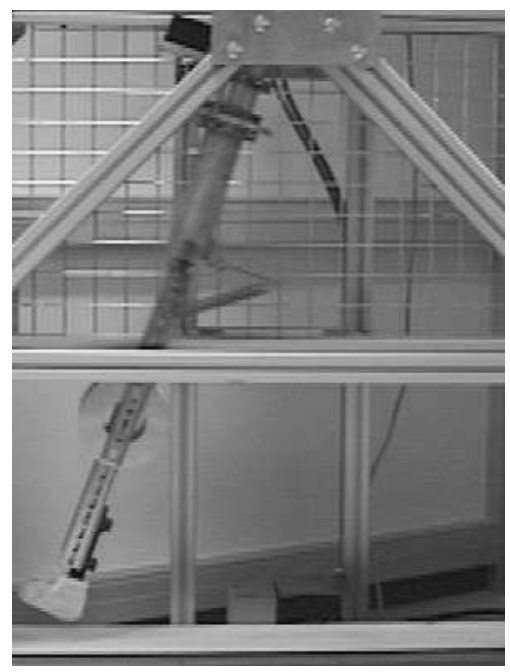

Figure 8: Step: Full extension 


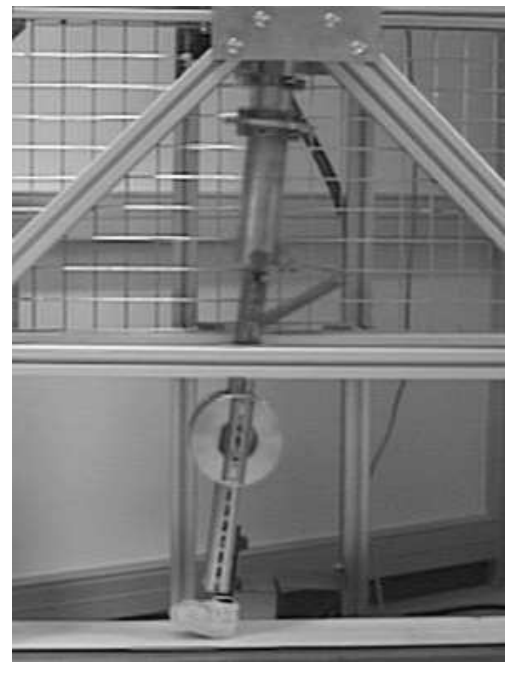

Figure 9: Step: Heel Contact

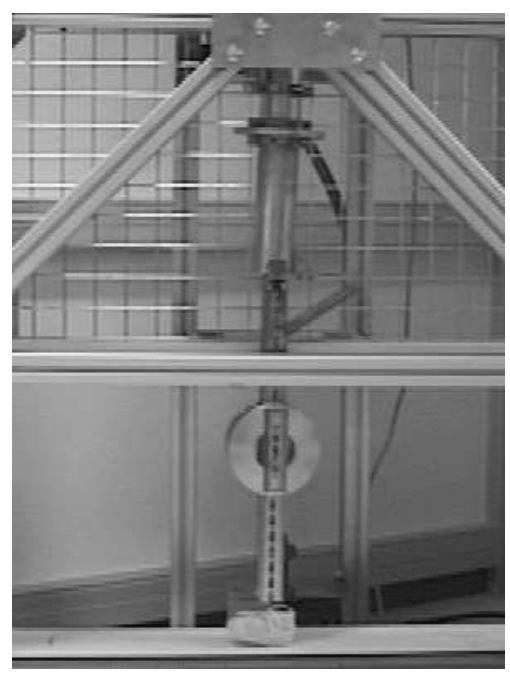

Figure 10: Step: Mid Stance

$\mathrm{RR} \mathrm{n}^{\circ} 3544$ 


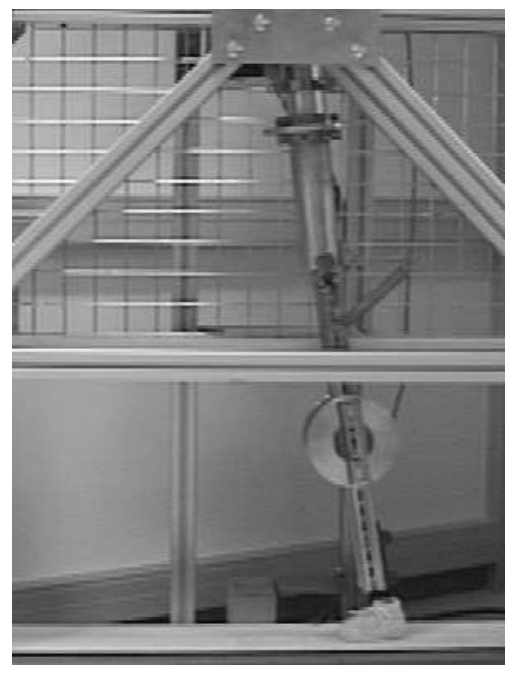

Figure 11: Step: End Stance

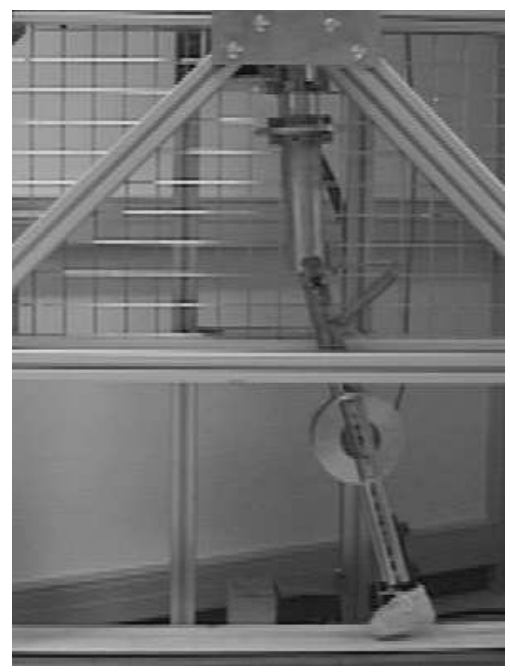

Figure 12: Step: Toe Off 


\section{Conclusion}

In this paper we have presented the analysis of a problem of ballistic "walking" and we have proposed a control scheme which has been validated experimentally. We intend now to use the experimental testbed for studying improvments of the approach, mainly in two directions: a first idea would be to specify the desired behavior as a limit cycle (as done in [20]) and to try to design a control allowing to stabilize it; a second possibility would be to specify only the structure of the control, its parameters having to be adapted from one step to another, through an iterative learning control approach. This last method would allow to cope with unknown physical parameters, like masses or stiffnesses, that we cannot presently do.

\section{References}

[1] P. Allard, M. Tuhaime, P.S. Thiry, G. Drouin: “ Use of Gait Simulation in the Evaluation of a Spring-loaded Knee Joint Orthosis for Duchène Nuscular Dystrophy Patients", Medical and BIological Engineering and Computing, vol 19, pp 165-170, 1981

[2] P. De Vita, M. Torry, K.L. Glover, D. L. Sperroni: “A Functional Knee Bracers Alters Joint Torque and Power Patterns during Walking and Running", J. Biomechanics, vol 29, no 5, pp 583-588, 1996

[3] I. Guigues: "Biomechanical Evaluation of a Device for Assistance in Knee Flexion", Master Thesis (in french), DEA STAPS, Université Joseph Fourier, Grenoble France, 1998

[4] R. McN. Alexander: "Three Uses for Springs in Legged Locomotion", The Int. J. of Robotics Research, 9(2), pp 53-61, 1990

[5] G.T. Yamaguchi, A. Takanishi: "Development of a Biped Walking Robot Having Antagonistic Driven Joints Using Nonlinear Spring Mechanisms", Proc. IEEE Int. Conf. on Robotics and Automation, pp 185-192, Albuquerque, New Mexico, USA, April 1997

[6] R.Q. van der Linde: "Active Leg Compliance for Passive Walking", Proc. IEEE Int. Conf. on Robotics and Automation, Leuwen, Belgium, May 1998

[7] M. Bergerman, C. Lee, Y. Xu: “ Experimental Study of an Underactuated Manipulator", Proc. TEEE/RSJ Int. Conf. on Intelligent Robots and Systems, pp 317-322, Pittsburgh, USA, Aug. 1995

[8] M. Bergerman, C. Lee, Y. Xu: “A Dynamic Coupling Index for Underactuated Manipulators", J. of Robotics Systems, vol 12, no 10, pp 693-707, oct. 1995

[9] S. Mochon, T.A. McMahon: "Ballistic Walking: An Improved Model", Mathematical Biosciences, 52, pp 241-260, 1980

RR n ${ }^{\circ} 3544$ 
[10] M. C. Laiou, A. Astolfi: "Underactuated Nonlinear Systems with Non-stabilizable Linear Approximation: A Case Study", IFAC Int. Workshop on Motion Control, pp 23-238, Grenoble, France, sept. 1998

[11] A. De Luca, R. Mattone, G. Oriolo: "Control of Underactuated Mechanical Systems: Application to the planar 2R Robot", Conference on Decision and Control, pp 1455-1460, Kobe, Japan, 1996

[12] M. Reyhanoglu, A. van der Shaft, N.H. McClamroch, I. Kolmanovsky: "Nonlinear Control of a class of Underactuated Systems", Conference on Decision and Control, pp 1682-1687, Kobe, Japan, 1996

[13] G. Oriolo, Y. Nakamura: "Control of Mechanical Systems with Second-order Nonholonomic Constraints: Underactuated Manipulators", Conference on Decision and Control, pp 2398-2403, Brighton, England, 1991

[14] G.A. Pratt, M.M. Williamson: "Series Elastic Actuators", Proc. IEEE/RSJ Int. Conf. on Intelligent Robots and Systems, pp 399-406, Pittsburgh, USA, Aug. 1995

[15] Pratt, J., Dilworth, P., Pratt, G.: “ Virtual Model Control of a Bipedal Walking Robot", Proc. IEEE Int. Conf. on Robotics and Automation, Albuquerque, New Mexico, USA, April 1997

[16] Spong, M.:" Swing Up Control of the Acrobot Using Partial Feedback Linearization", Prep. IFAC Symposium on Robot Control, pp 833-838, Capri, Italy, sept 1994

[17] M.D. Berkemeier, R.S. Fearing: "Sliding and Hopping Gaits for the Underactuated Acrobot", IEEE Trans. on Robotics and Automation, vol 14, no 4, pp 629-634, August 1998

[18] F. Saito, T. Fukuda, F. Arai: "Swing and Locomotion Control for a Two-link Brachiation Robot", IEEE Control Syst. Magazine, vol 14, pp 5-12, Feb. 1994

[19] J.J. Borrelly and al.: "The Orccad Architecture", The Int. J. on Robotics Research, vol 17, no 4, pp 338-359, April 1998

[20] A. Goswami, B. Espiau, A. Keramane: "Limit Cycles in a Passive Bipedal Gait and Passivity-mimicking Control Laws", Journal of Autonomous Robots, vol 4, pp 273-286, 1997

[21] B. Espiau, R. Boulic: "On the Computation and Control of the Mass Center of Articulated Chains", INRIA Research Report, no 3479 (http://www.inria.fr/RRRT/publications-eng.html), submitted to Multibody System Dynamics

[22] F. Génot, B. Espiau:"On the Control of the Mass Center of Legged Robots Under Unilateral Constraints", CLAWAR Symposium, Bruxelles, Nov. 1998 


\section{APPENDIX Proof of the Proposition}

It takes two steps:

1- Let us refer to equation (30). $M_{22}$ is always positive; $M_{12}$ is positive when $c_{2}>-\left(\frac{2 I_{2}}{m_{2} l_{2} l_{1}}+\right.$

$\left.\frac{l_{2}}{2 l_{1}}\right)$, which allows to define $\Theta$ as always greater than $\frac{\pi}{2}$. For example, take $I_{2}=\frac{m_{2} l_{2}^{2}}{4}$. If $l_{2}=l_{1}, M_{12}$ is always positive; if $l_{2}=l_{1} / 2, q_{2}$ has to belong to $\left[-\frac{2 \pi}{3}, \frac{2 \pi}{3}\right]$.

2- The zero dynamics part of (1) writes as:

$$
0=M_{12} \ddot{q}_{1}+M_{22} \ddot{q}_{2}+N(q, \dot{q})
$$

i.e.

$$
\ddot{q}_{2}=-\frac{M_{12} \ddot{q}_{1}}{M_{22}}-\frac{N}{M_{22}}
$$

The boundedness joint velocities implies that the Coriolis/centrifugal term is bounded. For geometric reasons, the gravity term and the force exerted by the spring knee as mounted are bounded. Therefore $N^{\prime}$ is bounded and the result is obvious.

$\mathrm{RR} \mathrm{n}^{\circ} 3544$ 
Unit'e de recherche INRIA Lorraine, Technopôle de Nancy-Brabois, Campus scientifique, 615 rue du Jardin Botanique, BP 101, 54600 VILLERS LÈS NANCY

Unit'e de recherche INRIA Rennes, Irisa, Campus universitaire de Beaulieu, 35042 RENNES Cedex Unit'e de recherche INRIA Rhône-Alpes, 655, avenue de l'Europe, 38330 MONTBONNOT ST MARTIN Unit'e de recherche INRIA Rocquencourt, Domaine de Voluceau, Rocquencourt, BP 105, 78153 LE CHESNAY Cedex Unit'e de recherche INRIA Sophia-Antipolis, 2004 route des Lucioles, BP 93, 06902 SOPHIA-ANTIPOLIS Cedex

Éditeur

INRIA, Domaine de Voluceau, Rocquencourt, BP 105, 78153 LE CHESNAY Cedex (France) http://www.inria.fr

ISSN 0249-6399 